\title{
Nutritional Status and Associated Factors Among Lactating Mothers in Nekemte Referral Hospital and Health Centers, Ethiopia
}

\author{
Temesgen Desisa Hundera ${ }^{1}$, Dessalegn Wirtu ${ }^{1}$, Habtamu Fekadu Gemede ${ }^{1,2, ~ *, ~}$ \\ Dunkana Negussa Kenie ${ }^{1}$ \\ ${ }^{1}$ Nutrition and Food Science, Wollega University, Nekemte, Ethiopia \\ ${ }^{2}$ Center for Food Science and Nutrition, Addis Ababa University, Addis Ababa, Ethiopia
}

Email address:

fekadu_habtamu@yahoo.com (H. F. Gemede),simbokom@gmail.com (H. F. Gemede)

\section{To cite this article:}

Temesgen Desisa Hundera, Dessalegn Wirtu, Habtamu Fekadu Gemede, Dunkana Negussa Kenie. Nutritional Status and Associated Factors Among Lactating Mothers in Nekemte Referral Hospital and Health Centers, Ethiopia. International Journal of Nutrition and Food Sciences. Vol. 4, No. 2, 2015, pp. 216-222. doi: 10.11648/j.ijnfs.20150402.23

\begin{abstract}
Lactating mothers from low-income countries including Ethiopia are considered as a nutritionally vulnerable group due to different socio demographic factors which impact on the health and wellbeing of children. Adequate nutrition for the mothers during lactation is therefore important for their good health as well as for that of their offspring. We aimed to assess nutritional status, and associated factors among lactating mothers in Nekemte Hospital and Health Centers, East Wollega, Ethiopia. Institutional based cross-sectional study was conducted from January 2014 to June 2014. Body Mass index was used to measure the nutritional status by measuring height and weight of the lactating mothers using standard procedures. Data on socio-demographic characteristics, maternal nutritional status, and associated factorswas collected from all lactating mothers who visited both health institutions during postnatal care and EPI program. The association of nutritional status with socioeconomic and demographic factorswere analyzed using descriptive statistics, binary and multiple logistic regression analysis. This study revealed that majority of the women 260(81.3\%) was in the age range of 17-25 yrs and attending school 292 (91.3\%). The prevalence of underweight, normal, overweight and Obesity were 65(20\%), 240(75\%), 20(4.7\%) and (0.3\%), respectively. From multiple logistic regression analysis family size (AOR=4.604, 95\%CI=1.903-11.140 andfamily income $(\mathrm{AOR}=0.250,95 \% \mathrm{CI}=0.100-0.623)$ were significantly associated with the nutritional status of the study participants. Nutritional statuses of the lactating mothers were short of the national and international recommendations. Therefore, sustained nutrition education is recommended to the lactating mothers and their families and communities to improve food intake, proper dietary knowledge during lactation in order to enhance health and nutrition outcomes of lactating mothers and their children. Proper family planning and the way that lactating mothers increase their income should be designed by concerned body.
\end{abstract}

Keywords: Lactating Mothers, Nutritional Status, Associated Factors

\section{Introduction}

Nutrition is the sum total of the processes involved in the intake and utilization of food substances by living organisms, including ingestion, digestion, absorption, transport and metabolism of nutrients found in food (Melvin , 2006); a fundamental pillar of human life, health and development throughout the entire life span. Proper food and good nutrition are essential for survival, physical growth, mental development, performance and productivity, health and a wellbeing of all living things (WFP, 2010)
Good nutrition is the cornerstone for survival, health and development for current and succeeding generations. However, the nutrition requirement varies with respect to age, gender and during physiological changes such as pregnancy, infant, lactating mothers and older age groups. Lactating mother was not only living for herself but for the infant, for the family and for the society and she carries great sole responsility in the family. Breastfeeding is the most precious gift a mother can give to her infant. When there is illness or malnutrition it may be a lifesaving gift. When there is poverty it may be the only gift. (Lawrence, 2001).

A mother's nutritional status at conception, during 
lactation, plays a key role in determining her health and wellbeing, as well as that of her child. So does the quality and quantity of her dietchild. The nutritional status of an individual is often the result of many inter-related factors. It is influenced by food intake, quantity \& quality, \& physical health. The spectrum of nutritional status spread from obesity to severe malnutrition .However, the nutrition requirement varies with respect to age, gender and during physiological changes such as pregnancy, infant, lactating mothers and older age groups. Nutrient requirements are considerably elevated during lactation than in any other stage of a woman's reproductive life.(Insel, 2003).

Nutritional status is an indication of the overall wellbeing of a population. It has been reported that lactation has different effects on maternal nutritional status depending on its duration, intensity, as well as cultural diversity (Rasmussenand Guire, 2004). In a cross sectional study (Sansui and Falana, 2009) it was reported that breastfeeding when practiced either exclusively or otherwise had no significant negative effect on the nutritional status of the mothers. Likewise, a longitudinal study (Ukegbu et al., 20012). Observed that irrespective of breastfeeding pattern, lactation also had no profound effect on maternal body Composition. However, the high energy cost of lactation as well as the nutritional and health risk it could pose for the woman emphasizes the need for continuous monitoring of their nutritional status and dietary intake in poor resource countries.

Lactating mothers and children are the most vulnerable to malnutrition due to: low dietary intakes \& low family income, inequitable distribution of food within the household, low educational level \& low occupational status, frequent round delivery $\&$ culture and work over load in the house hold, lack of frequent meal delivery \& low family encouragement, limited accessibility of nutritional information and nutritional knowledge, infectious diseases, and health care.If the mother is underweight during lactating the nutrients that are transferred to the baby will be of poor quality and quantity. On the other hand, if the mother is overweight, it will hamper the blood circulation to the uterus and restricts the quantity of nutrients transferred to the mammary gland of mothers for milk production and to the baby. Lack of nutrition in lactate mothers induce metabolic disturbances early in life of infancy, particularly those related to nutrition, induce irreversible physiologic alterations in infant. Therefore, nutritional inadequacy of the lactating mothers not only affects milk composition and production but also the health of the mothers, infancy and adulthood of human life (Sansui and Falana, 2009).

About 14 million adolescent girls worldwide become lactating mothers each year and more than $90 \%$ of these very young and reproductive mothers live in developing countries. The studies show that Ethiopia is one of the countries with the highest levels of lactating mothers malnutrition in SubSaharan Africa. Among malnutrition under nutrition is a serious problem in Ethiopia \& lactating mothers and children are the most affected segments of the population. One of every four lactate mothers in Ethiopia is undernourished (CSA, 2006).Information on nutritional status and associated factors of the lactating women are urgently needed for prioritizing, designing and initiating intervention programs aimed at improving maternal nutrition. Therefore, this study was aimed to assess the nutritional status, nutritional knowledge and associated factors of lactating mothers in the study area.

\section{Material and Methods}

\subsection{Study Design}

A cross sectional institution based study design was employed to assess nutritional status, and associated factors of lactating mothers in the study area.

\subsection{Source Population}

Lactating mothers living in Nekemte City who were visiting Nekemte Hospital and Health Centers for family planing and postnatal care during the study period care were considered as the source of population.

\subsection{Study Population}

Sampled lactating mothers were who visiting Nekemte Referral Hospitals and Health Centers for family planning and postnatal careduring data collection period.

\subsection{Inclusion Criteria}

Those lactating mothers (age 15-49) living in Nekemte City and who were visiting at Nekemte Hospital and Health centers for post natal care during the study period.

\subsection{Exclusion Criteria}

Lactating mothers who were critically ill, have hearing impairment and physical deformity during anthropometric measurements.

\subsection{Sample Size Determination}

The sample size of the study was determined by considering the country prevalence rate of under nutrition of lactating mothers (25\%) reported by CSA (2001). The expected prevalence rate the sample size was calculated with $5 \%$ marginal error and $95 \% \mathrm{CI}$ and none response rate of $9 \%$. Based on the above assumption, the sample size for the study was determined using the formula for single population proportion as follows.

Hence, the sample size (n) of lactate mothers was calculated as follow.

$$
\mathrm{n}=\frac{(Z \alpha / 2)^{2} \mathrm{p}(1-\mathrm{q})}{d^{2}}
$$

Where:

$\mathrm{n}=$ sample size

$\mathrm{Z}=\mathrm{Z}$ value corresponding to a $95 \%$ level of 
significance $=1.96$

$\mathrm{p}=$ expected prevalence rate of lactating $=25 \%=0.25$

$\mathrm{q}=(1-\mathrm{p})=(1-0.25)=0.75$

$\mathrm{d}=$ Absolute precession $(5 \%)$

$$
\mathrm{n}=\frac{1.96^{2} \times 0.25 \times 0.75}{0.05^{2}} \quad n=288+29=317
$$

Therefore, 288 lactating mothers of 15-49 years were examined for this particular study and in addition to none response rate of $9 \%(=32)$ lactating mothers.

\subsection{Sample Procedure}

The calculated sample size was proportionally allocated to the hospital and health centers based on their population size according to the average number of clients registered prior to the study period in the respective institutions.

\subsection{Data Collection Procedure}

A structured questionnaire first prepared in English and translated to Afan Oromo and then translated back to English to check its Consistency. The main points included in the questionnaire were used to assess the socio-demographic characteristics and the nutritional knowledge of the lactating mothers. Trained female research assistants who are fluent in the local language administered the pre-tested questionnaire to selected lactating mothers in the hospital, health centers and their own respective homes. For administering the structured questionnaire, four female nurses were recruited from the study area to conduct exit interview. Training was given for two days (including half day of pretest) on the objective, relevance of the study, confidentiality of information, respondent's right, informed consent and techniques of interview. Moreover, class room practical demonstration of the interview was carried out. Two Supervisors who has first degree in nursing have supervised the data collection. They closely followed up the data collection process throughout the data collection period along with the principal investigator. Data collected from the field were revised each night every day with data collectors and errors were corrected for the next day data collection.

\subsection{Anthropometric Measurements}

The nutritional status of the mothers was assessed using body mass index (Quetelet index). This indicator is the most frequently used standardized indicator of nutritional status to assess the progressive malnutrition in developing countries. It is defined as the weight in kilograms divided by the square of the height in meters $(\mathrm{kg} / \mathrm{m} 2)$. The cut-off point suggesting underweight in adults $(\mathrm{BMI}<18.5)$ was adopted from international Dietary Energy Consultative Group (James et al., 2008).

Measures of height in centimeters (to the nearest $0.1 \mathrm{~cm}$ ) and weight in kilograms to (the nearest $0.1 \mathrm{~kg}$ ) for every mother were taken using a weighing scale with an attached height meter (Seca) provided at the Nekemte hospital and health centers. These measures of height and weight were done no shoes and with light closing the mother facing away from the scale according to the standard protocol. The measures of height and weight were recorded on the questionnaire and were later used to calculate the Body Mass Index (BMI) of the mother.

\subsection{Socio-Demographic Variables}

This section comprised of 17 questions aimed at gathering background information and socio-economic status of the respondents. The questionnaire obtained data on age, religion, ethnicity, educational status, occupation, family size, marital status, husband's occupation, husband's educational level, family income, information about nutrition during breastfeeding. These variables were used to assess the nutritional status of the lactate mothers.

\subsection{Data Quality Assurance}

Quality issues were addressed through the following measures to ensure the data generated is complete, reliable, accurate and above all reproducible. These measures contribute towards both internal and external validity of the study. To ensure the quality of data, training of data collectors and supervisors were undertaken on $5 \%$ of the total sample size to assess its clarity, length, completeness and consistency. The questionnaire is also translated in to local language to facilitate more understanding of the respondents.

Supervisors and principal investigator closely supervised the data collection process. Filed questionnaires were checked daily for completeness and errors were corrected. Meetings were held to address problems and clarify issues that could hamper collection of good data with data collectors. Checking for completeness and accuracy of completed data was done at the end of each day of data collection.

\subsection{Data Analysis}

The data was cheeked, cleared, entered into computer and analyzed using SPSS software for windows version 20 . Descriptive statistics was computed to determine the proportions, percentages, frequency distribution. Both bivariate and multivariate analyses are employed to identify the determinants of nutritional status of lactating mothers. These analyses focus onoutcomes of nutritional status and for lactating mothers whether they are undernourished or not. Since the interest is in identifying lactating mothers at risk of malnutrition, the dependent variables are coded as 1 lactating mothers is undernourished and coded as 0 if not.The bivariate analysis, was employed to see the association between each of the independent and dependent variables under study and the nutritional status of lactate mothers as measured by underweight and p-values less than 0.05 are considered as significant. The chi-square bivariate analysis doesn't consider confounding effects; therefore, the net effects of each independent variable are estimated controlling other factors using the logistic regression multivariate analysis.

The odds ratio, which is determined from the logistic 
regression coefficients, tells us the increased or decreased chance of malnutrition given a set level of the independent variable while controlling for the effects of the other variables in the model. Estimates of odds greater than 1.0 indicate that the risk of malnutrition is greater than that for the reference category. Estimates less than 1.0 indicate that the risk of malnutrition is less than that for the reference category of each variable.

To identify associated factors, first a bivariate logistic regression was performed for each independent variable with the outcome of interest [Nutritional Status (BMI).Finally, multivariable logistic regression was done to determine independent predictors of nutritional Status (BMI).. All tests were two-sided and $p<0.05$ was considered statistically significant

\section{Result}

\subsection{Socio-Economic Characteristics of the Respondents}

Table 1. Socio economic characteristics observation in each variable.

\begin{tabular}{|c|c|c|}
\hline Variables & Categories & Frequency(n) \\
\hline \multirow{4}{*}{ Age groups } & $17-25$ & $260(82 \%)$ \\
\hline & $26-35$ & $40(12.6 \%)$ \\
\hline & $36-49$ & $20(6.3 \%)$ \\
\hline & Total & $317(100 \%)$ \\
\hline \multirow{5}{*}{ Marital status } & Married & $290(91.4 \%)$ \\
\hline & Single & $25(8 \%)$ \\
\hline & Widowed & $3(0.9 \%)$ \\
\hline & Divorced & $2(0.6 \%)$ \\
\hline & Total & $317(100)$ \\
\hline \multirow{5}{*}{ Level of education } & Illiterate & $28(8.8 \%)$ \\
\hline & 4-Jan & $55(17.4 \%)$ \\
\hline & 12-May & $117(36.6 \%)$ \\
\hline & Diploma and above & $120(37.8)$ \\
\hline & Total & $317(100 \%)$ \\
\hline \multirow{6}{*}{$\begin{array}{l}\text { Occupational status } \\
\text { of Mothers }\end{array}$} & Employed & $57(17.9 \%)$ \\
\hline & Un employed & $48(15.1 \%)$ \\
\hline & Business & $29(9.1 \%)$ \\
\hline & Housewife & $175(55.2 \%)$ \\
\hline & Others & $17(5.3 \%)$ \\
\hline & Total & $317(100 \%)$ \\
\hline \multirow{5}{*}{ Family size } & $1-2$ & $63(19.8 \%)$ \\
\hline & $3-5$ & $119(37.5 \%)$ \\
\hline & $6-7$ & $94(29.6 \%)$ \\
\hline & $>7$ & $44(13.8 \%)$ \\
\hline & Total & $317(100 \%)$ \\
\hline \multirow{6}{*}{$\begin{array}{l}\text { Occupational status } \\
\text { of } \mathrm{H}\end{array}$} & Governmental employers & $140(44.1 \%)$ \\
\hline & Merchant & $89(27.5 \%)$ \\
\hline & Farmers & $35(11 \%)$ \\
\hline & Daily workers & $26(8.2 \%)$ \\
\hline & Others & $30(9.4 \%)$ \\
\hline & Total & $317(100 \%)$ \\
\hline \multirow{5}{*}{ Monthly income } & $>2000$ birr & $137(43.2 \%)$ \\
\hline & $1999-1000$ birr & $58(18.2 \%)$ \\
\hline & $999-500$ birr & $72(22.7 \%)$ \\
\hline & $<500$ birr & $53(16.7 \%)$ \\
\hline & Total & $317(100 \%)$ \\
\hline
\end{tabular}

Table1 in presents the percentage distribution of lactating mothers age (15-49) according to the selected demographic and socio-economic characteristics. The response rate was $100 \%$. The majority of the respondents, $292(88.2 \%)$, were in the age range of less than 30 years.By ethnicity, more than three fourth, $258(81.3 \%)$ of the lactating mothers were Oromo. Regarding religion fifty percent of them, 161 (50.7\%), were Protestant, 93 (29.3\%) were Orthodox and 66 (20.8\%) were Muslims. Most of thestudy participants, 290 $(91.4 \%)$, were married.

Regarding educational status, the majority, 292 (91.3\%), have ever attended school. 117(36.6\%) of the lactating mothers, attended from junior to high school. While, 120 $(37.8 \%)$ of them have learned up to diploma and above. Concerning occupational status more than half, 175 (55.2\%) were housewives and about one-fifth, 57 (17.9\%), were government employed. With regard to family size, 119 (37.5\%), 63 (19.8\%), and 94 (29.6\%) have 1-2, 3-5 and 6-7 children respectively. The majority of the husbands, 140 (44.1\%), were government employees and some of them, 89(27.8), were working on their own business. Regarding their monthly income, out of the total interviewed lactating mothers, $137(43.2 \%)$ were getting 2000 birr per month while $53(16.7 \%)$ are getting 500 or less Ethiopian Birr.

\subsection{Nutritional Status of Lactating Mothers}

The nutritional status of lactating mother was measured by Body Mass Index (BMI), weight in kilogram divided by height square in meter, based on WHO standard classification. For the majority of participants, $240(75 \%)$, BMI was in the normal range, that is from $18.5-24.9 \mathrm{~kg} / \mathrm{m}^{2}$. About one-fifth of the respondents, $65(20.5 \%)$ have BMI of less than 18.5 $\mathrm{kg} / \mathrm{m}^{2}$, whereas $15(4.7 \%)$ and $5(1.5 \%)$ have BMI greater than or equal to $25 \mathrm{~kg} / \mathrm{m}^{2}$ respectively (table 2 ).

Table 2. Anthropometric measurement of lactating mothers in Nekemte, East Wollega, 2014.

\begin{tabular}{lll}
\hline BMI, $\mathbf{~ k g} / \mathbf{m}^{2}$ & Classification of BMI & Frequency $\%$ \\
\hline$<18.5$ & Underweight & $65(20.5 \%)$ \\
$18.5-24.9$ & Normal weight & $235(74.1 \%)$ \\
$24.9-29.9$ & Overweight & $15(4.7 \%)$ \\
$>30$ & Obese & $5(1.5 \%)$ \\
\hline
\end{tabular}

\subsection{Associated Factors of Nutritional Status of Lactating Mothers}

To determine the association between nutritional status and explanatory variables, bivariate analysis was performed using logistic regression model. The results were shown in Table 3. The result indicatedthat there wasassociation between nutritional status of lactating mothers and some of the explanatory variables under the study. As shown in the table occupation of mother, family income, family size, husband educational status and definition of foods were found to be associated with nutritional status of lactating mother. 
Table 3. Bivariate logistic regression test of nutritional status of lactating mothers with selected variables 2014.

\begin{tabular}{|c|c|c|c|c|c|}
\hline \multirow{2}{*}{ Variable } & \multirow{2}{*}{ Categories } & \multicolumn{2}{|l|}{ BMI, $\mathrm{kg} / \mathrm{m}^{2}$} & \multirow{2}{*}{$P$-value } & \multirow{2}{*}{ COR $(95 \% \mathrm{CI})$} \\
\hline & & underweight $(<18.5)$ & Normal weight $(\geq 18.5)$ & & \\
\hline \multirow{4}{*}{$\begin{array}{l}\text { Husbands } \\
\text { education }\end{array}$} & Illiterate & $4(25 \%)$ & $12(75 \%)$ & 0.05 & $0.590(0.175-1.991)$ \\
\hline & Primary(1-4) & $13(34.2 \%)$ & $25(65.8 \%)$ & 0.043 & $0.378(0.1690 .846)^{*}$ \\
\hline & Junior to high school (5-12) & $33(26.2 \%)$ & $93(73.8 \%)$ & 0.023 & $2.554(1.3052 .007)^{* *}$ \\
\hline & Diploma and above & $23(16.4 \%)$ & $117(83.6 \%)$ & 0 & 1 \\
\hline \multirow{9}{*}{$\begin{array}{l}\text { Mother } \\
\text { Occupation }\end{array}$} & employed & $26(38.3 \%)$ & $40(61.7 \%)$ & 0.9 & $1.842(0.703-4.828)$ \\
\hline & Un employed & $41(62 \%)$ & $32(48 \%)$ & 0.015 & $11.508(2.25258 .794)^{* *}$ \\
\hline & Business & $2(12.9 \%)$ & $27(87.1 \%)$ & 0.064 & $1.883(0.691-5.130)$ \\
\hline & House wife & $80(25.3 \%)$ & $112(74.7 \%)$ & 0.002 & $4.35(1.044-17.672)^{* *}$ \\
\hline & Others & $7(38.9 \%)$ & $11(61.1 \%)$ & 0.048 & 1 \\
\hline & $>2000$ birr & $20(13.7 \%)$ & $126(86.3 \%)$ & 0.005 & $0.412(0.104-0.430)^{*}$ \\
\hline & $1999-1000$ birr & $10(18.5 \%)$ & $44(81.5 \%)$ & 0.007 & $0.376(0.184-0.768)^{*}$ \\
\hline & 999 - 500 birr & $19(29.7 \%)$ & $45(70.3 \%)$ & 0.598 & $0.698(0.304-1.607)$ \\
\hline & $<500$ birr & $24(42.9 \%)$ & $32(57.1 \%)$ & 0.057 & 1 \\
\hline \multirow{4}{*}{ Family size } & 2-Jan & $13(27.1 \%)$ & $35(72.9 \%)$ & 0.53 & $1.671(0.702-3.976)$ \\
\hline & 5-Mar & $28(27.7 \%)$ & $73(72.3 \%)$ & 0.013 & $1.618(0.778-3.364)$ \\
\hline & 7-Jun & $14(11.3 \%)$ & $110(88.7 \%)$ & 0.000 . & $4.877(2.171-10.957)^{* *}$ \\
\hline & $>7$ & $18(38.3 \%)$ & $29(61.7 \%)$ & 0.006 & 1 \\
\hline
\end{tabular}

Husband educational status, definition of food and family income of lactating mothers are weakly associate to the outcome variables $(\mathrm{p}<0.01)$, but occupation of mother and family size of lactating mothersare strongly associated with nutritional status $(\mathrm{p}<0.001)$. Whereas, other predictable variables like age, marital status, culture, ethnicity, religion, husband occupation, and nutritional information have no association with nutritional status of lactating mothers $(\mathrm{p}>0.05)$. Concerning husband education, those mothers whose husband has at leastprimary education were less likely develop under weight than those husband who has no formal education $\quad(\mathrm{COR}=0.378,95 \% \mathrm{CI} \quad=0.169-0.846)$. Family income is another factor found to affect the nutritional status. Family oflactate mothers those who got monthly income greater than 2000 birr and 1999 - 1000 birr were less likely exposed underweight than those who gotless than 500 birr $(\mathrm{COR}=0.412,95 \% \mathrm{CI}=0.104-0.430) \quad$ and $\quad(\mathrm{COR}=0.376$, $95 \% \mathrm{CI}=0.184-0.768)$ respectively. More over family size of lactate mothers were another determinant factor that affects nutritional status. Those mothers who have family size of greater than three children were more likely exposed to underweight than who have less family sizein the house hold $(\mathrm{COR}=4.877,95 \% \mathrm{CI}=2.171-10.957)$.

After bivariate analysis, multivariate analysis of logistic regression was performed to filter the net effect of each independent variable that associate in the bivariate model analysis with nutritional status of lactate mothers by controlling the other independent variablesin table 4 . In a multivariate logistic regression analysis family size, family income and definition of food were found to have significant association with nutritional status of lactate mothers. Concerning family income, those lactating mothers earnmonthly income greater than 2000 birr were less likely to be under weight than those lactating mothers who got less than 500 birr $(\mathrm{AOR}=0.250,95 \% \mathrm{CI}=0.100-0.623)$. Those lactate mothers who have the concept of food were less likely to be under weight than those mothers who have no sufficient information about food (AOR $=0.521,95 \% \mathrm{CI}=0.280-0.970)$. On the other hand, mothers who have family sizemore than three were 4.6 times more likely exposed to underweight than those lactating mothers who have less family size. $(\mathrm{AOR}=4.604,95 \% \mathrm{CI}=1.903-11.140)$.

Table 4. Multiple logistic regression of nutritional status of lactate mother.

\begin{tabular}{|c|c|c|c|c|c|}
\hline \multirow{2}{*}{ Variable } & \multirow{2}{*}{ Categories } & \multicolumn{2}{|c|}{ Nutritional status of Lactating mothers } & \multirow{2}{*}{ P-Value } & \multirow{2}{*}{$\operatorname{AOR}(95 \% \mathrm{CI})$} \\
\hline & & Underweight $\left(<18.5 \mathrm{~kg} / \mathrm{m}^{2}\right)$ & Normal weight $\left(\geq 18.5 \mathrm{~kg} / \mathrm{m}^{2}\right)$ & & \\
\hline \multirow{4}{*}{ Family income } & $>2000$ birr & $20(13.7 \%)$ & $126(86.3 \%)$ & 0.007 & $0.250(0.100-0.623)^{*}$ \\
\hline & $1999-1000$ birr & $10(18.5 \%)$ & $44(81.5 \%)$ & 0.045 & $0.446(0.193-1.026)$ \\
\hline & 999 - 500 birr & $19(29.7 \%)$ & $45(70.3 \%)$ & 0.071 & $0.874(0.339-2.254)$ \\
\hline & $<500$ birr & $24(42.9 \%)$ & $32(57.1 \%)$ & 0.028 & 1 \\
\hline \multirow{4}{*}{ Family size } & $1-2$ & $13(27.1 \%)$ & $35(72.9 \%)$ & 0.93 & $1.65(.634-4.317)$ \\
\hline & $3-5$ & $14(11.3 \%)$ & $110(88.7 \%)$ & 0.003 & $4.60(1.903-11.140)^{*}$ \\
\hline & $6-7$ & $28(27.7 \%)$ & $73(72.3 \%)$ & 0.002 & $5.92(3.858-4.307)^{* *}$ \\
\hline & $>7$ & $18(38.3 \%)$ & $29(61.7 \%)$ & 0.001 & 1 \\
\hline
\end{tabular}




\section{Discussion}

\subsection{Effect of Socio Demographic Factors on Nutritional Status}

This study assessed the level of nutritional status and associated factors in Nekemte town, East Wollega, Ethiopia. The nutritional status of lactating mother was measured by Body Mass Index (BMI), weight in kilogram divided by height square in meter, based on WHO standard classification. Out of the total participants measurement had taken most of them were in the normal BMI range 240(75\%), that is from $18.5-24.9 \mathrm{~kg} / \mathrm{m}^{2}$.One fifth of the study participants $65(20 \%)$ had BMI less than $18.5 \mathrm{~kg} / \mathrm{m}^{2}$ which were underweight. Whereas fifteen $(4 \%)$ of participants were overweight with BMI greater than $25 \mathrm{~kg} / \mathrm{m}^{2}$ and $5(1 \%)$ of them had BMI of greater than thirty which were obsess. This indicates that the prevalence of underweight was significant which was found tobe similar with the finding of Darussalam, 21.2\% underweight, (WFP and UNICEF, 2010). This is may be attributed to the impact of socio -demographic factors such as family income, family size, husband education, occupation status of mother and nutritional information which may contributed to the incidence of dietary inadequacies. On the other hand compared to similar study in Mekele, Ethiopiathe underweight in Nekemte was lower, $25 \%$ (Haileslassie K et al., 2013).

This study revealed that family income has a due effect on the nutritional status of lactate mother. Those mothers who have monthly family income greater than 1000 birr were less likely exposed to underweight. This finding is similar with the UNICEF study on lactate mothers (UNICEF, 2010). This indicates that household economic status is positively associated with maternal nutrition status, which is a prerequisite for access to adequate dietary intake and improved nutritional status for members of maternal nutritional status.In developing countries, it has been reported that the income of a household is an important determinant of its access to food which in turn is a major determinant of child and maternal nutrition and wellbeing (Miller $\mathrm{N}$ et al., 2005]. Epidemiological studies have reported on the relationship between diet and poverty; and the report established that the purchasing power of a family indicates the level of household food security and types of diets that are ultimately consumed by household members (Kirna S, 2005).

According to this study finding another important factor that influence nutritional status of lactate mother was family size Those lactating mothers whose family size was greater than six (6) were found to be more likely to develop underweight which is similar to the study conducted in Accra, Ghana in family size was predictor of nutritional status of mothers (Amanuelet al., 2013).This may be associated with risk factors such as mothers' inadequate capacity for caring her children. The mother herself may be biologically depleted from too frequent births, and this could also negatively affect the nutritional status of the lactate mothers and newborn baby as a result of the intergenerational link. Therefore, access to services for child spacing could benefit the youngest child and the mother. Prolonging the intervals between births, through increasing demand for family planning and/or fulfilling unmet need for family planning, could be important elements of strategies to improve lactating mothers nutrition status.

Occupational is also another important socioeconomic variable explaining nutritional status. According this study, unemployment or unpaid (cash) employment of women was a significant factor for nutritional status of mothers as compared with mothers employed for cash. House wife and unemployed mothers were more vulnerable to under nutrition than business owners or other occupation mothers. The probable reason for this could be that House wife and unemployed mothers workers have lower educational status than mothers working employed (EDHS, 2005) and they have relatively less decision-making autonomy and less control over income than women with governmental employers. Women's paid employment could provide an additional income source that can improve food security of the household and raise the status of women by allowing them to have more control over resources. Some evidence also indicates that the nutritional impact of increased household income is a function of the income earner and the kind of income (ACC/SCN, 2003). This founding was similar with that unemployed women were at high risk of under nutrition, even in households with a relatively better socioeconomic status .The same result has been observed among other sub-Saharan countries (Hoffman DR,2004).

On the hand husband education and nutritional information are other factors that significantly associate to nutritional status of lactate mother. Regarding husband education, those families whose husband had minimum of primary education were less likely exposed to under nutrition. Similar study conducted inMalaysia demonstrated that individuals with better nutritional knowledge levels are significantly higher in educational level, nutritional attitude and occupational status. The finding of the study stated that individuals with a higher educational level had better nutrition knowledge and higher nutrition knowledge of employed women in study may be explained by more access to internet, books and magazines as source of information in work area (Melkieet al., 2005).

\subsection{Determinants of Nutritional Status of Lactating Mother}

As can be seen in the multivariate logistic regression analysis identified family income, family size and definition of food as the most determinant factors of nutritional status of lactating mother. The sample showed that, as compared to low monthly income $(<1000$ birr) family the high monthly income family lactating mothers were almost 0.25 less likely vulnerable to under nutrition. The sample also indicated that, the likelihood of being under nutrition was 4.6 and 5.9 times higher among lactating mothers who had 3-5 and 6-7 family size respectively. The study also showed that lactating mothers who have knowledge of food were less likely develops under nutrition. 


\section{Conclusion}

The present finding uncovered that low income lactate mothers have the highest rates of underweight. Family size and family income significantly affect nutritional status of lactating mothers. Other predictable variables like age, marital status, culture, ethnicity, religion, husband occupation, and nutritional information have no association with nutritional status of lactating mothers. Nutritional statuses of the lactating mothers were short of the national and international recommendations. Therefore, sustained nutrition education is recommended to the lactating mothers and their families and communities to improve food intake, proper dietary knowledge during lactation in order to enhance health and nutrition outcomes of lactating mothers and their children. Proper family planning and the way that lactating mothers increase their income should be designed by concerned body.

\section{References}

[1] Administration Committee on Coordination Subcommittee on Nutrition (ACC/SCN, 2003). Assessment of Nutritional Status in Emergency-Affected Population. Geneva, Switzerland.

[2] Amanuel R., Yimer H., yaikob K., (2013). Mother's Knowledge on Nutritional Requirement of Infant and Young Child Feeding in Mekelle, Ethiopia

[3] Central Statistical Authority (CSA) [Ethiopia] and ORC Macro, (2006).

[4] Hoffman DR., (2004).Maturation of visual acuity is accelerated in breast-fed term infants fedbaby food containing DHA-enriched eggs; Journal of Nutrition, 134: 2307- 2313.
[5] Insel P., Turner E., and Ross D.,(2003). Discovering nutrition: Jones and Bartlett, Thompson Wads worth, USA.

[6] Kinra S., Nelder RP., Lewendon GJ., (2005). Deprivation and childhood obesity: a cross sectional study ofMaternal nutrition and pregnancy outcomes, 4, 36-39.

[7] Lawrence M., and Lawrence R., (2001). Given the benefits of breastfeeding, what Contraindications exist?; Pediatric Clinics of North America, 48(1), 3(2), 55.

[8] Melkie Edris, Habtemariam Tekle, Yohannis Fitaw, Baye Gelaw, Dagnew Engedaw, Tigest Alemu, (2005).Maternal nutrition for the Ethiopian health center team, ), Addis Ababa, Ethiopia.

[9] Melvin W.H., (2006). Nutrition for Health, Fitness and Sport: 7th edition Mc Graw-Hill New York. pp9

[10] Muller O., Krawinkel M., (2005). Malnutrition and health in developing countries, CMAJ173:279-86.

[11] Rasmussen KM., and Guire KM., (20004). Effects of breastfeeding on maternal health and results from a randomized controlled trial of an educational intervention; American Journal of Results. Addis Ababa: United Nations Population Fund (UNFPA).

[12] Sanusi R., Falana O., ( 2009). The nutritional status of mothers practicing breastfeeding, 8, 46-59.

[13] Ukegbu PO., Waeg U., Bute AC., (20012). Body composition changes among lactating mothers

[14] United Nations Children's Fund, (2010). Infant and Young Child feeding response Available from: http://www.unicef.org/nutrition/index_24819.html accessed on 12th October.

[15] WFP, (2010). Special report on Crop and Food Security Assessment Mission to Ethiopia: Integrating the Crop and Food. 